\title{
Immediate fasting blood glucose response to electroacupunc- ture of ST36 versus CV12 in patients with type 2 diabetes mellitus: randomized controlled trial
}

\author{
ALI MOHAMED ALI ISMAIL ${ }^{1, A, E, F}$, ALSHAYMAA SHAABAN ABD EL-AZEIM², B-D \\ ORCID ID: 0000-0003-1447-8817 \\ ${ }^{1}$ Department of Physical Therapy for Cardiovascular/Respiratory Disorder and Geriatrics, Faculty of Physical \\ Therapy, Cairo University, Giza, Egypt \\ ${ }^{2}$ Basic Science Department, Faculty of Physical Therapy, Cairo University, Giza, Egypt
}

A - Study Design, B - Data Collection, C - Statistical Analysis, D - Data Interpretation, E - Manuscript Preparation, F - Literature Search, G - Funds Collection

Summary Background. Electroacupuncture (EA) is one of the main alternative or complementary therapies used to treat diabetic symptoms, but the immediate hypoglycemic effect of EA at one acupoint only has not been studied in type 2 diabetes mellitus (T2DM) patients.

Objectives. This study aimed to investigate the immediate response of fasting blood glucose (FBG) to EA at ST36 (Zusanli) versus CV12 (Zhongwan) acupoints in T2DM patients.

Material and methods. With a body mass index $<30 \mathrm{~kg} / \mathrm{m}^{2}$, one hundred T2DM men 40-73 years of age were randomly divided into four groups: a real EA of CV12 group, placebo-controlled EA of CV12 group, real EA of ST36 group and placebo-controlled EA of ST36 group. All groups - containing 25 T2DM men - received one session of a 30-minute 2-Hz EA. Pre- and post-FBG levels were evaluated for every patient within each group.

Results. The only group that showed a significant reduction of FBG was the real EA of CV12 group $(p<0.05)$ while the placebo-controlled EA of CV12, real EA of ST36 and placebo-controlled EA of ST36 groups did not reveal any significant FBG decline ( $p>0.05)$ in men with T2DM.

Conclusions. A 30-minute 2-Hz real EA of CV12 produced a highly significant decrease of FBG levels in T2DM men.

Key words: blood glucose, electroacupuncture, type 2 diabetes mellitus, randomized controlled trial.

Ismail AMA, El-Azeim ASA. Immediate fasting blood glucose response to electroacupuncture of ST36 versus CV12 in patients with type 2 diabetes mellitus: randomized controlled trial. Fam Med Prim Care Rev 2021; 23(4): 437-441, doi: https://doi. org/10.5114/fmpcr.2021.110370.

\section{Background}

In 2019, there were 8.9 million registered diabetic adults 20-70 years of age in Egypt, which represents the $9^{\text {th }}$ country with the highest documented number of diabetic patients worldwide. The 2013-estimated prevalence of diabetes mellitus (DM) was 7.5 million, and in 2035, it is expected to be 13.1 million [1].

DM is a group of metabolic disorders defined as hyperglycemia originating from defects in the secretion or action of insulin or both [2]. Besides the general positive effects of exercise on the psychological and physical sense of wellbeing in many patients [3], exercise is considered one of the therapeutic procedures for controlling/regulating plasma glucose levels in type 2 diabetes mellitus (T2DM) patients, in addition to hypoglycemic medications and different diet regimens [4].

Besides the long-term need for exercise to control T2DMasociated hyperglycemia [5], available hypoglycemic drugs are efficacious, but many pharmacological hypoglycemic agents can lead to serious complications and side effects [4]. Recently, with rapid medical advancement, complementary or alternative therapies have seen more attention from many patients due to the absence of complications and/or side effects, in addition to the hopeful results in the short- and long-term management of T2DM symptoms [6].
In Traditional Chinese Medicine (TCM), acupoint therapy, alone and/or combined with western medicine, is considered a main treatment for DM and/or its complications, with lower-reported rates of side effects than with the sole use of western medicine. Acupoint therapy is a treatment conducted through the meridians and acupoints utilizing one of the following: acupuncture, injection of acupoint, moxibustion, acupressure and external application [7].

For 2000 years, with positive evidence-based clinical effects in human and animal studies [8], alternative therapies have been used safely with no complications $[9,10]$ for T2DM treatment with different techniques of needle acupuncture stimulation [8]. Electroacupuncture (EA), a recent used clinical technique for needle stimulation, other than traditional manual hand stimulation, is a non-invasive technique [11] that uses a charge of electricity to enforce needle stimulation inserted into the selected meridians and points of acupuncture [12]. As it utilizes an electrical current that passes through the inserted acupuncture needles into single or multiple acupoints, EA is said to be a strong hypoglycemic treatment modality in patients with T2DM [13].

Based on the meridian theory, the Zusanli or Stomach 36 (ST36) acupoint, located at the stomach meridian under the knee within the tibialis anterior muscle [14], is commonly used as a general acupuncture acupoint to relieve symptoms of DM [7], restore the normal aliment of gastrointestinal functions, re- 
cover a general sense of well-being [15], refresh the functions of internal organs, including the pancreas [16], energize physical activities and cure the diseases [4].

Based on Korean medicine, the Zhongwan or Conception Vessel acupoint 12 (CV12) (located on the wall of abdomen) is a treatment point for disorders of the digestive organs, including the pancreas [17]. Studies have reported a significant hypoglycemic effect of manual acupuncture at a single acupoint with one needle inserted at CV12 [18] and ST36 [19] in T2DM patients.

Despite the evidence-based hypoglycemic effect of single-acupoint laserpuncture at BL 20 [20] and EA at GB 26 [21], ST36 [4] and CV12 [7] in diabetic rats, no published data at present - to our knowledge - has investigated the hypoglycemic effect of EA at ST36 or CV12 in T2DM patients.

With recommendations to investigate the immediate response of fasting blood glucose (FBG) to a 30 -minute single-acupoint EA at ST36 [19] or CV12 [18], this study was the first aimed at making a comparison between the immediate FBG response to a 30-minute EA at CV12 versus ST36 in T2DM men.

\section{Material and methods}

In addition to a signature obtained from all patients on the consent form for participation in the study, this randomized placebo-controlled prospective study followed the recommendations of the Helsinki Declaration. Under number P.T.REC/012/002919, the Local Scientific Ethical Committee for Human Research, Faculty of Physical Therapy, Cairo University, approved the carrying out of this study. Under identification number PACTR202010486385289, the trial was also registered with the Pan-African Clinical Trials Registry (PACTR).

\section{Sample size}

The number of patients was determined according to a pilot study on 20 patients with T2DM and G*Power (version 3.1.9.2) (Franz Faul, Uni Kiel, Germany). The calculation depended upon the F-test, power at $85 \%$ and type I error at $5 \%$, and the effect size of FBG was (0.32). The proper minimum sample size for this study was 92 patients.

\section{Participants}

With a body mass index $(\mathrm{BMI})<30 \mathrm{~kg} / \mathrm{m}^{2}$ and a FBG $>126$ $\mathrm{mg} / \mathrm{dl}$, one hundred T2DM men 40-73 years of age were randomly selected from the DM outpatient clinics of Cairo University, Om El-Masryeen, and Meetghamr General Hospitals during the period from 18 October to 30 November 2020.

Via a physician, patients with respiratory, renal, hepatic and cardiac disorders were excluded. Besides smokers and those with addictions, patients with hypertension, a cardiac pacemaker, malignancy, active dermatological infection, mental disorders and a history of admission with any type of complementary therapies within the last 3 months were also rejected from participation in this study.

The patients were divided into one of the four following groups (every group contained 25 men): group 1 (real-EA of CV12 group), group 2 (placebo-controlled EA of CV12 group), group 3 (real-EA of ST36 group), group 4 (placebo-controlled EA of ST36 group).

Figure 1 presents the consort flow diagram of the study. Utilizing a computer-generated randomized block list, a physiotherapy assistant - blinded to the trial protocol and not involved in any part of the treatment or assessment - divided the patients to one of the treatment groups.

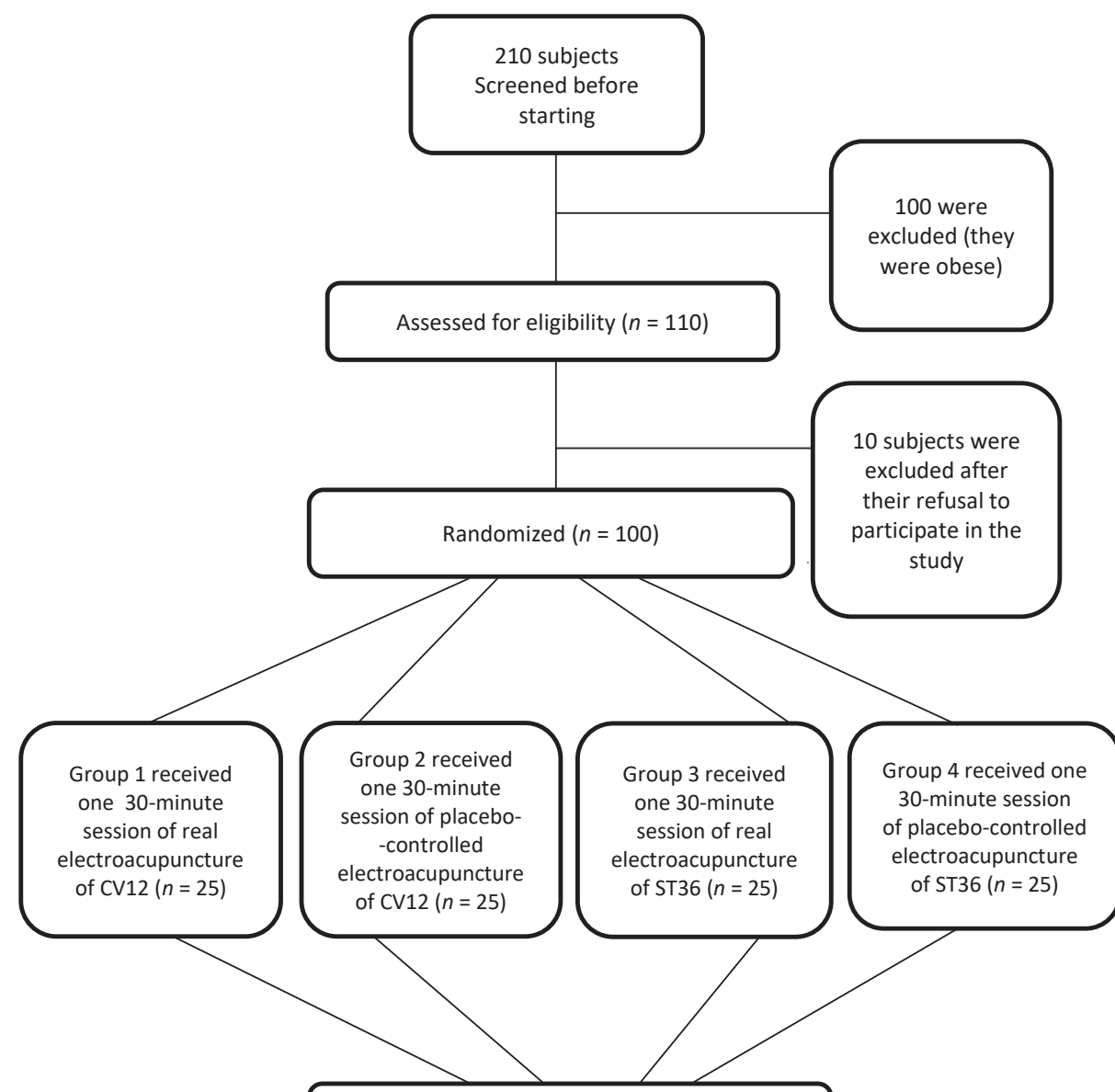




\section{Interventions}

\section{Group 1}

After insertion of the needle at the CV12 acupoint, which was located on the median anterior line of the upper abdomen, 4 cun (breadth of the thumb) proximal to the umbilical center [22], the de qi sensation was confirmed by all patients of this group via the manipulation of the needle into the CV12 acupoint; the needle then was connected to the EA device.

Group 2

Where there was no known acupoint at this area, the needle was placed one cun lateral to the CV12 acupoint in the right abdominal side in this group; the needle was then connected to the EA device.

Group 3

After insertion of the needle at the ST36 acupoint, which was located one cun lateral to the lower border of the tuberosity of tibia [23], the de qi sensation was confirmed by all patients of this group via the manipulation of the needle into the ST36 acupoint; the needle was then connected to the EA device.

\section{Group 4}

Where there was no known acupoint at this area, the needle was placed at the midline connecting the patellar apex and the tuberosity of tibia in this group; the needle was then connected to the EA device.

A sterile single-use stainless-steel acupuncture needle (CE 0197, made in China, with a size $0.25 \times 25 \mathrm{~cm}$ ) was inserted perpendicularly with a 0.5 -cun depth into the detected point in each group. In every patient within each group, the positive pole of the EA device (inter-TENS 668, made in Egypt) was connected to the needle, and the reference (negative) pole was fixed with a bandage one centimeter proximal to the needled point [23]. All patients within each group received only one session of 30-minute 2-Hz EA between 8 am to $9 \mathrm{am}$.

With overnight fasting, FBG was measured immediately before and after the session with every patient in all groups using a blood glucose meter (On Call Plus, made in China).

\section{Statistical analysis}

The collected data was handled using version 18 of the SPSS program (Chicago, SPSS Inc. IBM Corp.). After verification with the Kolmogorov-Smirnov normality test, the normal distribution of all data was confirmed. All baseline and post-treatment data among groups was treated using the one-way ANOVA test. To test the significant changes between pre- and post-FBG values within each group, multiple pairwise comparisons by a post hoc test was used. The significance value was determined at $p<0.05$.

\section{Results}

Regarding the baseline data (Age and BMI), Table 1 presents a non-significant difference among the four groups $(p>0.05)$. tPost tost Post tost Post tost Post tost Post tost Post tost The post hoc test (Table 2) revealed a significant difference between preand post-treatment at $\mathrm{G} 1$ as $p<0.0001$, while the other groups did not show any significant difference. Partial Eta Square was used to determine the effect size between groups post-treatment and found a large difference: $\eta 2=0.29$ (Table 2).

\section{Discussion}

The present study found a highly significant immediate decline of FBG after one session of a 30-minute real CV12-EA, while the placebo-controlled CV12-EA, real ST36-EA and placebo-controlled ST36-EA did not produce the same effect in T2DM men.

There is little literature that explains the mechanism of an immediate significant FBG drop after a 30-minute real-EA session at CV12, but studies on diabetic animal models justified this drop due to the increased production of endogenous B-endorphin [4] and pancreatic opioid peptides [24] that promote insulin production and sensitivity [4].

Stimulation of the real acupoints is guaranteed to produce a higher hypoglycemic effect than needle stimulation of adjacent non-acupoints [4]. Based on TCM, when a specific acupoint is needled, the effect of treatment occurs on the corresponding body part [25]. Greater insulin sensitivity is encouraged by the closest location of CV12 to the pancreas rather than the furthest location from the pancreas - ST36 (located in the upper anterolateral lower leg) [26].

The study was supported by Chang et al. [26], who found a non-significant hypoglycemic effect after a 30-minute EA session at bilateral ST36 in rats with induced DM. Non-significant changes within the placebo group [18] supported the present results, especially after a significant decrease of random blood glucose in the group that received one session of a 30-minute manually-acupunctured CV12 in T2DM patients. In accordance with the present results, another study revealed a non-significant reduction of FBG after 30-minute 2-Hz EA of bilateral ST36 in patients with DM-complicated gastric dysrhythmia [27].

Mohanty et al. [28] have contradicted the results of this study, as the 20-minute manual real-CV12 needling produced a mild non-significant hypoglycemia may be due to the very small number of participated healthy volunteers $(n=18)$. 30-minute bilateral manual needling at ST36 also produced a more effective significant reduction of random blood glucose levels in T2DM patients than the placebo group [19].

\begin{tabular}{|c|c|c|c|c|c|c|}
\hline & G1 & G2 & G3 & G4 & $F$ & $p$ \\
\hline Age (years) & $53.64 \pm 8.05$ & $54.28 \pm 8.23$ & $55.32 \pm 8.84$ & $52.64 \pm 8.25$ & 0.45 & $0.72^{\circ}$ \\
\hline BMI $\left(\mathrm{kg} / \mathrm{m}^{2}\right)$ & $28.46 \pm 1.22$ & $27.62 \pm 1.53$ & $28.06 \pm 1.37$ & $27.80 \pm 1.34$ & 1.74 & $0.16^{\circ}$ \\
\hline
\end{tabular}

G - group, BMI - Body Mass Index, kg - kilogram, $\mathrm{m}$ - meter, $p$-value significance was set at $<0.05$, ${ }^{\mathrm{a}}$ no significant difference between the four groups.

Table 2. Comparison between pre- and post-FBG within each group by post hoc test and between groups by One-Way ANOVA

\begin{tabular}{|c|c|c|c|c|c|c|c|}
\hline Outcome & G1 & G2 & G3 & G4 & $P$ & $\boldsymbol{F}$ & $\eta 2$ \\
\hline \multicolumn{8}{|l|}{$\mathrm{FBG}(\mathrm{mg} / \mathrm{dL})$} \\
\hline Pre-treatment & $140 \pm 11.87$ & $139.24 \pm 9.41$ & $138.84 \pm 8.63$ & $139.64 \pm 10.52$ & $0.98^{a}$ & 0.06 & \\
\hline Post-treatment & $124.92 \pm 8.49$ & $138.04 \pm 9.44$ & $137.44 \pm 8.78$ & $139.52 \pm 10.16$ & $0.0001^{b}$ & 13.38 & 0.29 \\
\hline$p$-value (within group) & $0.0001^{b}$ & $0.42^{a}$ & $0.35^{a}$ & $0.94^{a}$ & & & \\
\hline$\%$ of improvement & $10.7 \%$ & $0.86 \%$ & $1 \%$ & $0.08 \%$ & & & \\
\hline
\end{tabular}

$\mathrm{G}$ - group, FBG - fasting blood glucose, $\mathrm{mg}$ - milligram, dL - deciliter, $p$-value significance was set $<0.05$, ${ }^{\mathrm{a}}$ non-significance difference, ${ }^{\mathrm{b}}$ significance difference, Partial Eta Square - effect size (small > 0.01, medium >0.06, large >0.14). 
In opposition to the results of this study, in normal rats, EA with a frequency of $2 \mathrm{~Hz}$ at the ST36 produced a greater hypoglycemic response than stimulation at the CV12 acupoint [29]. A $15 \mathrm{~Hz}$ bilateral EA of ST36 improved prednisolone-induced insulin resistance and activity in male rats via the enhanced recovered expression of signal proteins of insulin in addition to the decreased levels of plasma-free fatty acids [13].

\section{Limitations of the study}

The absence of a follow-up, lack of female inclusion and the large number of diabetic patients in each group are the main limitations of this study.

\section{Conclusions}

A 30-minute 2-Hz elctro-acupuncture of CV12 - within the limitations of this study - produced a highly significant decline of FBG levels in men with T2DM.

Source of funding: This work was funded from the authors' own resources.

Conflicts of interest: The authors declare no conflicts of interest.

\section{References}

1. Ismail AMA, Eld E, Abdel Aal MEM. Impact of transcutaneous electrical nerve stimulation (TENS) on hyposalivation in type 2 diabetics. Biosci Res 2019; 16(1): 690-694.

2. Ismail AMA, Abd El-Azeim AS. Short-term intraocular pressure response to the combined effect of transcutaneous electrical nerve stimulation over acupoint (Acu-TENS) and yoga ocular exercise in type 2 diabetic patients with primary open-angle glaucoma: a randomized controlled trial. J Acupunct Meridian Stud 2021; 14(5): 193-199.

3. Ismail AMA, Tolba AMN, Felaya EEE. Effect of aerobic exercise training on leptin and liver enzymes in non-diabetic overweight hepatitis C men. Advances in Rehabilitation 2021; 35(2): 17-23, doi: 10.5114/areh.2021.106027.

4. Lee YC, Li TM, Tzeng CY, et al. Electroacupuncture at the Zusanli (ST-36) acupoint induces a hypoglycemic effect by stimulating the cholinergic nerve in a rat model of streptozotocine-induced insulin-dependent diabetes mellitus. Evid Based Complementary Altern Med 2011; 2011: 650263, doi: 10.1093/ecam/neq068.

5. Zhou C. Nursing intervention on exercise therapy for patients with type 2 diabetes mellitus complicated with peripheral vascular disease. Acta Microsc 2020; 29(1); 439-446.

6. Ismail AMA, Abd Elfatah Abo Saif HF, El-Moatasem AMM. Effect of Jyoti-Trataka on intraocular pressure, autonomic control, and blood glucose in diabetic patients with high-tension primary open-angle glaucoma: a randomized-controlled trial. J Complement Integr Med 2021, doi: 10.1515/jcim-2021-0041.

7. Chang SL, Lin JG, Chi TC, et al. An insulin-dependent hypoglycaemia induced by electroacupuncture at the Zhongwan (CV12) acupoint in diabetic rats. Diabetologia 1999; 42(2): 250-255, doi: 10.1007/s001250051146.

8. Chen C, Liu J, Sun M, et al. Acupuncture for type 2 diabetes mellitus: a systematic review and meta-analysis of randomized controlled trials. Complement Ther Clin Pract 2019; 36: 100-112, doi: 10.1016/j.ctcp.2019.04.004.

9. Ismail AMA, Abdelghany Al, Elfahl AMA. Immediate effect of interscapular cupping on blood pressure, oxygen saturation, pulse rate and chest expansion in sedentary smoker students. J Complement Integr Med 2021; 18(2): 391-396, doi: 10.1515/jcim-2020-0050.

10. Cheng $\mathrm{H}$, Zhao L, Ju Z, et al. Effects of 10.6- $\mu \mathrm{m}$ laser moxibustion and electroacupuncture at ST36 in a 5-Fu-induced diarrhea rat model. Supportive Care Cancer 2020; 1-9, doi: 10.1007/s00520-020-05788-0.

11. Lin W, Jia D, Fu C, et al. Electro-acupuncture on ST36 and SP6 acupoints ameliorates lung injury via sciatic nerve in a rat model of limb ischemia-reperfusion. J Inflamm Res 2020; 13: 465, doi: 10.2147/JIR.S264093.

12. Lee CH, Kim DK, Yook TH, et al. Effectiveness of electroacupuncture at Zusanli (ST36) on the immunohistochemical density of enteroendocrine cells related to gastrointestinal function. J Acupunct Meridian Stud 2012; 5(2): 63-71, doi: 10.1016/j.jams.2012.01.002.

13. Lin RT, Tzeng CY, Lee YC, et al. Acute effect of electroacupuncture at the Zusanli acupoints on decreasing insulin resistance as shown by lowering plasma free fatty acid levels in steroid-background male rats. BMC Complement Altern Med 2009; 9(1): 1-9, doi: $10.1186 / 1472-6882-9-26$.

14. Wang LQ, Chen Z, Zhang K, et al. Zusanli (ST36) acupoint injection for diabetic peripheral neuropathy: a systematic review of randomized controlled trials. J Altern Complement Med 2018; 24(12): 1138-1149, doi: 10.1089/acm.2018.0053.

15. Lu Y, Ji B, Zhao G, et al. Comparison of protective effects of electroacupuncture at ST36 and LU5 on pulmonary and hypothalamic pituitary adrenal axis changes in perinatal nicotine-exposed rats. Biomed Res Int 2020, 2020: 3901528, doi: 10.1155/2020/3901528.

16. Rousdy A. Effectiveness of acupressure at the Zusanli (ST-36) acupoint as a comfortable treatment for diabetes mellitus: a pilot study in Indonesia. J Acupunct Meridian Stud 2017; 10(2): 96-103, doi: 10.1016/j.jams.2016.12.003.

17. Kim MS, Sung B, Ogay V, et al. Novel circulatory connection from the acupoint Zhong Wan (CV12) to pancreas. J Korean Pharmacopunct Inst 2008; 11(1): 13-19, doi: 10.3831/KPI.2008.11.1.013.

18. Kumar R, Mooventhan A, Manjunath NK. Immediate effect of needling at CV-12 (Zhongwan) acupuncture point on blood glucose level in patients with type 2 diabetes mellitus: a pilot randomized placebo-controlled trial. J Acupunct Meridian Stud 2017; 10(4): 240-244, doi: 10.1016/j.jams.2017.06.003.

19. Mooventhan A, Ningombam R, Nivethitha L. Effect of bilateral needling at an acupuncture point, ST-36 (Zusanli) on blood glucose levels in type 2 diabetes mellitus patients: a pilot randomized placebo controlled trial. J Complement Integr Med 2020; 7(3): 20190100, doi: 10.1515/jcim-2019-0100.

20. Cornejo-Garrido J, Becerril-Chavez F, Carlin-Vargas G, et al. Antihyperglycaemic effect of laseracupuncture treatment at BL20 in diabetic rats. Acupunct Med 2014; 32: 486-494, doi: 10.1136/acupmed-2014-010573.

21. Li YY, Hu H, Liang CM, et al. Effects of electroacupuncture stimulation of "Daimai" (GB 26) on body weight, blood glucose and blood lipid levels in rats with metabolism syndrome. Zhen Ci Yan Jiu 2014; 39: 202-206.

22. Gao $L$, Chen $B$, Zhang $Q$, et al. Acupuncture with different acupoint combinations for chemotherapy-induced nausea and vomiting: study protocol for a randomized controlled trial. BMC Complement Altern Med 2016; 16(1): 441, doi: 10.1186/s12906-016-1425-1.

23. Chen B, Hu SX, Liu BH, et al. Efficacy and safety of electroacupuncture with different acupoints for chemotherapy-induced nausea and vomiting: study protocol for a randomized controlled trial. Trials 2015; 16(1): 212, doi: 10.1186/s13063-015-0734-x.

24. Peplow PV, Baxter GD. Electroacupuncture for control of blood glucose in diabetes: literature review. J Acupunct Meridian Stud 2012; 5(1): 1-10, doi: 10.1016/j.jams.2011.11.018. 
25. Gergess SH, Hesham AAR, Ghada S, et al. Effect of electroacupuncture on glucostasis in diabetic rats. Med J Cairo Univ 2018; 86: 3589-3596.

26. Chang SL, Tsai CC, Lin JG, et al. Involvement of serotonin in the hypoglycemic response to $2 \mathrm{~Hz}$ electroacupuncture of zusanli acupoint (ST36) in rats. Neurosci Lett 2005; 379(1): 69-73, doi: 10.1016/j.neulet.2004.12.057.

27. Chang $\mathrm{CS}, \mathrm{Ko} \mathrm{CW}, \mathrm{Wu} \mathrm{CY}$, et al. Effect of electrical stimulation on acupuncture points in diabetic patients with gastric dysrhythmia: a pilot study. Digestion 2001; 64(3): 184-190, doi: 10.1159/000048860.

28. Mohanty S, Mooventhan A, Manjunath NK. Effect of Needling at CV-12 (Zhongwan) on blood glucose levels in healthy volunteers: a pilot randomized placebo controlled trial. J Acupunct Meridian Stud 2016; 9(6): 307-310, doi: /10.1016/j.jams.2016.08.002.

29. Chang SL, Lin JG, Hsieh CL, et al. Comparison of hypoglycemic effect in different acupoints response to $2 \mathrm{~Hz}$ electroacupuncture. J Chin Med 2002; 13(2): 111-117.

Tables: 2

Figures: 1

References: 29

Received: 22.02 .2021

Reviewed: 24.03 .2021

Accepted: 16.09 .2021

Address for correspondence:

Ali Mohamed Ali Ismail, PhD

Department of Physical Therapy for Cardiovascular/Respiratory Disorder and Geriatrics

Faculty of Physical Therapy

Cairo University

Giza

Egypt

Tel.: 0201005154209

E-mail: ali.mohamed@pt.cu.edu.eg 